\title{
Postpartum Green Star Family Planning Decision Aid for Pregnant Adolescents in Tanzania: a Qualitative Feasibility Study
}

\author{
Stella Emmanuel Mushy ( $\nabla$ pendostellam@gmail.com ) \\ Muhimbili University of Health and Allied Sciences https://orcid.org/0000-0002-4867-2709 \\ Eri Shishido \\ St. Luke's International University \\ Sebalda Leshabari \\ Muhimbili University of Health and Allied Sciences \\ Shigeko Horiuchi \\ St. Luke's International University
}

Research

Keywords: decision aid, postpartum family planning, long-acting reversible family planning, intrauterine copper device, implants, rapid repeat pregnancy, adolescent mothers, pregnant adolescents, Tanzania

Posted Date: March 3rd, 2021

DOI: https://doi.org/10.21203/rs.3.rs-265412/v1

License: @ () This work is licensed under a Creative Commons Attribution 4.0 International License. Read Full License

Version of Record: A version of this preprint was published at Reproductive Health on August 9th, 2021. See the published version at https://doi.org/10.1186/s12978-021-01216-6. 


\section{Abstract}

Background: The use of a decision aid (DA) in clinical settings has been useful as they inform and educate clients about the available options that help them to reduce decision-making conflict related to feeling uninformed and unclear about personal values compared with usual care. There is a dearth of published data about the use of decision aid during family planning (FP) counseling with postpartum women focusing on long-acting reversible family planning methods. Therefore, the researchers developed a decision aid named "Green Star" and assessed its feasibility. The study outcomes were practicality, usefulness, and acceptability as perceived by the pregnant adolescents and the nurse/midwives.

Methods: An exploratory in-depth qualitative interview involving six nurse/midwives with three or more years of experience in FP services, and 12 pregnant adolescents aged 15-19 years. Purposive sampling was used to select the participants, and sampling relied on the saturation principle of data collection. Researchers used a semi-structured interview guide translated into Kiswahili language. Data were transcribed and analyzed following inductive-deductive content analysis.

Results: The length of the presentation was found to be just right, with time ranging from twenty minutes to one hour. The flow of the information was stated to be good with small significant changes suggested. Kiswahili language used was reported to be appropriate and well elaborated, however, a few words were suggested to be rephrased to reduce ambiguity. The nurse/midwives reported that the DA included most of the important information clients wanted to know during FP counseling. The adolescents stated that the DA improved their knowledge and provided new information about the existence of FP immediately after childbirth. The participants said the DA clearly addressed the benefits and side effects as well as dispelled some myths and misconceptions. The DA was accepted to be useful in complementing the daily family planning counseling offered as well as in improving adolescents' knowledge.

Conclusion: The postpartum Green Star family planning decision aid was perceived to be practical, useful, and acceptable. Further research is needed to assess the effect of the DA in the choice for postpartum long acting reversible FP among the pregnant adolescents in Tanzania.

\section{Plain English Summary}

This study assessed the practicality, usefulness, and acceptability of the postpartum Green Star family planning decision aid for pregnant adolescents. The study was conducted at Amana district hospital in Dar es Salaam, Tanzania. Study participants, six nurse/midwives with three or more years of experience in family planning (FP) services, and 12 pregnant adolescents aged 15-19 years, were recruited for indepth interviews using a semi-structured interview guide with five questions. Both the pregnant adolescents and the nurse/midwives felt that the decision aid in Kiswahili included important information women desire to know during FP counseling and that the length of the information was just right, ranging from 20 minutes to one hour. It was recommended that the nurse/midwives should implement the decision aid to ensure comprehensibility of the presented information and pictures.

The decision aid was perceived to be useful in improving knowledge, clearly addressing several myths and misconceptions as well as the benefits and side effects of each option. Several changes were recommended: the flow of the information needed reorganization and some Kiswahili words needed rephrasing to reduce ambiguity. Furthermore, the study participants accepted the decision aid to be used in the clinical settings as it had evidence-based information useful to compliment the FP counseling offered especially for non-permanent FP methods. The decision aid was said to improve the objectivity of the FP counseling as well as impart knowledge about the existence of FP methods offered immediately after childbirth.

\section{Background}

Adolescent pregnancies remain a global public health concern in the world, with the highest rate happening in developing countries. Across the world, each year, about 16 million girls aged 15-19 years are estimated to give birth [1], and the majority of the pregnancies are reported to be unplanned and unwanted [2].

According to the Tanzania Demographic Health Survey [3], the adolescent population (10-19) comprised $23 \%$ of the total population (44.9 million). Most adolescents are sexually active before the age of 15 years old and the number of childbearing adolescents aged 15-19 years has gradually increased from $23 \%$ in 2010 to $27 \%$ in 2016. Likewise, the adolescent fertility rate has increased from 116 to 132 per 1,000 girls between 2010 and 2016, and at the age of 19 years, almost $56.7 \%$ of the adolescents have begun childbearing.

Adolescent pregnancies remain a significant national problem and critical health and social priority in Tanzania $[4,5]$. One-quarter $(24 \%)$ of the pregnancies among young women are reported to be unwanted [6]. Country statistics show that $21 \%$ of the young women's pregnancies 
are among adolescents aged $15-19$ years whereby $18.1 \%$ are first births, and $2.9 \%$ are second or later births [3].

Most women who start childbearing in their teenage years have more children, and shorter birth spacing. Furthermore the majority of their births were reported as unwanted compared to women started childbearing when they were 20 years or older [7]. Subsequent pregnancies during teen years compound the economic, physical, and psychological consequences of adolescent mothers [7, 8]. Failure to complete high school and impaired economic sufficiency are significantly associated with repeated pregnancy during adolescent age [9].

Inconsistent use of contraception, lack of knowledge about immediate use of family planning after birth, lack of communication between the teen and her health care providers, fear of side effects, and myths and misconceptions about FP have been reported to increase the risk of adolescent pregnancy and rapid repeat pregnancies (RRP) [10-12]. Weight gain, reduced sexual desire, irregular bleeding, infertility, and abnormal vaginal discharge are the main barriers cited that hindered female youth from going for FP services [10].

To prevent early pregnancies, countries across the world including Tanzania have employed sexual and reproductive health education, negotiation skills-building, and improving awareness and accessibility to modern contraceptives among adolescents [13]. However, most of the interventions focus on the prevention of the first pregnancies with fewer efforts on the prevention of subsequent unwanted pregnancies during the teen years. As a result, most adolescents continue to experience RRP despite the availability and accessibility of FP methods. The majority (78\%) of non-first pregnancies among adolescent mothers in Tanzania, experience RRP within three months after birth, with over half of births (51\%) occurring in the second year from last birth [12]. This is an alert where researchers, healthcare providers, and policymakers should put more efforts to help pregnant adolescents (PAs) prevent RRP as it compounds the existing challenges faced during teen pregnancy.

Delaying the second birth will provide a better chance for an adolescent mother to mature physically, psychologically, and biologically. Her opportunities to finish high school, plan for the future, and develop other training skills are increased [14]. Additionally, risks of premature births, stillbirths, and newborn morbidity and mortality will be reduced [15].

Effective use of FP is one of the primary and essential strategies for reducing high-risk pregnancies that often occur too early and too frequently [16]. Use of long-acting reversible family planning (LARFP) immediately after childbirth, i.e. intrauterine copper devices (IUCDs) and implants, are high-impact interventions to avoid the risks associated with adolescent pregnancy [17]. The methods do not rely on daily, weekly, or monthly use, and have higher continuation and satisfaction rates compared with short-acting contraceptives [17]. A review study on the effects of the LARFP on RRP in adolescents found that adolescent mothers who initiated LARFP methods after delivery had a lower risk of RRP as compared with those who adopt shorter-acting ones or no FP methods [18].

Despite significant gains in the training of healthcare providers, FP commodity distribution, and quality of care, still, PPFP uptake among adolescent mothers is critically underutilized; only $12.2 \%$ of the adolescent mothers used PPFP within three months after delivery, the largest proportion using injectables followed by pills [3]. Both IUCDs and implants are extremely effective in preventing pregnancy, and they all last for several years and are easy to use [1, 17]. For these reasons, pregnant adolescents need comprehensive information about each option to help them decide on the contraceptive they want as there is no universal "best choice," just a matter of clarifying the values and beliefs individuals have about LARFP methods.

Evidence shows that the use of decision aids in clinical settings has been useful as they inform and educate clients about the available options that help them to reduce decisional conflict related to feeling uninformed and unclear about personal values compared with usual care [19]. Recently, decision aids are widely used to provide health information to both clients and the public [20]. Since the IUCDs and implants have almost equal effectiveness and they are long lasting, PA's choices will depend on their preferences and lifestyle. The PAs must clarify their values by appraising the benefits and harms of each option and weighing attributes that are personally important to them when making a choice.

While the effect of the decision aids (DAs) on FP counseling have been evaluated and found to have a positive outcome [21, 22], according to researcher's knowledge, no study has used a decision aid that focuses on LARFP methods to improve FP uptake by adolescent mothers immediately after childbirth. To avoid the long term physiological, psychological, and economic consequences adolescent mothers face from unwanted RRP as a result of non-contraception use, the researchers developed a decision aid named the postpartum Green Star. The "Green Star" name was borrowed from Kiswahili word "Nyota ya Kijan" and is well known by most people in the country born before 1992. When FP was launched in the country in 1992, the word "Nyota ya Kijani" was used as a symbol of the FP project. The name was used to sensitize women of reproductive age to use artificial FP methods to space and limit the number of children couple should have. The postpartum Green Star family planning decision aid included updated evidence-based information on IUCDs and implants that are believed to help guide PAs in the process of making decisions on the best option for their preference about LARFP. This study focused on assessing 
the practicality, usefulness, and acceptability of the decision aid as perceived by pregnant adolescents (PAs) and healthcare providers (HCPs) in the choice for PPFP among PAs.

\section{Materials And Methods}

\section{Study design}

The study used an exploratory qualitative research design. In August 2020, we conducted 18 in-depth interviews that involved the HCPs and PAs from Amana district hospital in Dar es Salaam. Though the DA was designed for use by the PAs; we included the HCPs because we wanted to hear their opinions about the DA. The researchers believed that the FP counseling experiences the HCPs had would help the researchers get significant and well-informed comments to improve the contents in the decision aid as well as the intervention process.

\section{Study population, recruitment, and data collection process}

The study population consisted of $6 \mathrm{HCPs}$ and 12 PAs. The HCPs were nurses/midwives with three or more years of experience in FP services while the PAs were aged 15-19 years who were able to read and communicate in the Kiswahili language and consented to participate in the study. The study's principal investigator $(\mathrm{PI})$ and the person in charge of the maternity clinic were responsible for recruiting the study participants who were purposively selected to best answer the research questions. The interviews lasted for 40-50 minutes and were led by two moderators: one who asked the questions assisted by another who recorded the interview and took notes. A semi-structured interview guide with five questions in the Kiswahili language was used to collect information from the PAs and the HCPs. To assess the practicality, usefulness, and acceptability of the postpartum Green Star family planning decision aid, participants were asked: "What do you say about the information presented in the decision aid? How do you feel about the use of this decision aid for making a decision about postpartum family planning? What do you say about the usefulness of this decision aid? Will you recommend its use in the clinic? And several follow-up questions.

Ethical clearance was acquired from St. Luke's International University, Muhimbili University of Health and Allied Sciences, and the National Institute of Medical Research. A study permission from the regional medical officer and the medical officer in charge of the Amana hospital was likewise acquired. The interviews were conducted in a private room within the hospital to ensure convenient access and privacy. The PI informed the participants about the purpose, scope, and importance of the study at the beginning of each discussion. Study participants were also informed of their rights to participate and withdraw at any time during the interview whenever they felt like it. The researchers obtained written informed consent from each participant for their participation in the interview and recording. The PI requested the participants to feel free and open when responding to questions and assured them that there was no right or wrong answer. The PI and a research assistant (RA) conducted the interviews. Experience in conducting interviews, experience in using recorders, having knowledge, and experience in FP were the criteria used to select the RA. A one-day training for the RA was conducted on the purpose of the study, what questions to ask and how to ask and probe, as well as when to obtain written informed consent from the participants so as not to influence the process of data collection.

\section{Data processing and analysis}

The PI uploaded the audio files into a secured computer with a passcode immediately after all interviews each day. The interviews were directly transcribed verbatim in Kiswahili language and the data was analyzed while in Kiswahili language. Thematic analysis was conducted following the steps outlined by Braun and Clarke [23]. An iterative-deductive, team-based coding approach was employed to code and analyze the data [24]. The PI and RA who conducted the in-depth interviews undertook the coding process and analysis. Individual codes were then organized into sub-categories and categories. Study participants' quotes illustrated the key findings,

The PI and RA, using a team-based approach, developed the codebook [24] after re-reading all the transcripts (familiarization with data). The PI and RA had several meetings where codebooks and memos were presented, codes updated and any existing disagreement resolved. This iterative process continued until discrepancies were resolved and an agreement was reached on the meaning of codes and their applications of all questions in all 18 transcripts.

Next the PI and RA generated themes that involved open-ended coding of several transcripts with no predetermined codes or categories. Coding was done directly onto the hard copies of the transcripts during multiple readings of the interviews. Independent from each other, the PI and RA coded interviews question by question, and then shared and compared their individual findings of the coding with the purpose of reconciling differences, if any. The PI and RA applied the codes from the codebook to all 18 transcripts. Codes were refined, reduced, and expanded during this period. Concerns and disagreements were addressed and reconciled immediately during this phase in a 
continuous process until agreement on the codes and definitions was reached. Finally, the PI and RA generated larger categories and subcategories based on the findings of the initial coding. Codes were grouped under these larger findings of categories, and sub-categories.

\section{Development of the postpartum Green Star family planning decision aid}

A thorough literature review of previous studies was carried out and the gap, target population, and the objectives were identified to address the research problem. The researchers mainly focused on previously published studies to determine the objectives to be addressed [21, 22, 25-27]. There was a dearth of publications that used decision aid in reducing decision making conflict on the utilization of LARFP methods.

The researchers then identified the individual needs of the PAs participants by reviewing a previous study that looked at the barriers to the utilization of FP among female youths in Dar es Salaam, Tanzania [10]. The individual needs of the participants included inadequate knowledge, especially on the LARFP methods as female teens showed to have several misbeliefs about the methods, as well as how to participate in decision making as the majority could not decide on their own without involving their sexual partners.

The content, design, and arrangement of the DA prototype development were grounded in the Ottawa Patient Decision Aid Development eTraining (ODAT) [28], International Patient Decision Aid Standards Collaboration checklist [29], Theory of Planned Behavior [30], Health Belief Model [31], Social Cognitive Theory [32], current clinical guides for FP counseling for providers [1, 33], and findings from previous studies on benefits and side effects of the options, satisfaction and continuation rates, and fertility return [34-40]. The prototype DA has four components based on the Ottawa Patient Decision Aid development guide: i) know how to make a decision with conviction; ii) understand the characteristics of the decision; iii) clarify what is important to you and iv) make the decision. The prototype DA was then shared with a team of three experts that included a research supervisor with several years of experience in maternal and child health as well as experience in developing decision aids, a midwife with decision aid developing experience, and another midwife with both teaching and clinical experience in maternal and child health including FP. The aim of sharing the DA with these experts was to receive comments on the comprehensibility and usefulness of the DA, which the researchers were able to incorporate to modify the decision aid. The final DA version 1 was given to 6 HCPs and 12 PAs to assess its practicality, usefulness, and acceptability (Figure 1).

\section{Results}

\section{Socio-demographic characteristics of the study participants}

A total of 18 participants were interviewed; 6 HCPs (2 male and 4 female midwives) and 12 PAs. One HCP had a bachelor's level of education while the rest had a master's level. All HCPs had FP service experience of a minimum of three years (3-12 years of experience). The majority (83\%) of the PAs had a primary level of education, more than $58.3 \%$ had no employment, and half of them were still single. More than half $(66.7 \%)$ of the PAs reported the index pregnancy to be their first pregnancy.

The present study looked at the practicality, usefulness, and acceptability of the postpartum Green Star family planning decision aid in the choice for postpartum family planning among PAs in Tanzania from both adolescents and HCPs. From the data analysis, we will present three major categories with sub-categories as derived from the questionnaire: 1) practicality, 2) usefulness, and 3) acceptability.

\section{Practicality}

The study participants were asked to discuss their views about the information presented in the decision aid (length i.e., too long, or too short or just right, and quality of the presented information in terms of flow, clarity, and comprehensibility of the information and language used) in relation to the level of education of PAs with a mainly primary level of education. The findings are as follows:

\section{a) Right length of the information presented}

The PAs and the HCPs said the length of the presentation was okay i.e., just right. However, the HCPs reported the amount of time required to reading the DA varied from 20-50 minutes, and the concern was the reading speed and understanding ability of the participants that are perceived to be different.

"By looking, the details are not too long or too short. I found that she can spend 50 minutes if she is a slow reader and if she is a fast reader then 20 minutes will be enough" [HCP]

The minimum time reported by the PAs to finish reading the DA was 25 minutes, while the maximum time was 60 minutes.

"It took me 20-30 minutes to read the whole DA" [adolescent] 


\section{b) Good quality of the information}

The study participants were asked to give their views about the flow of information, clarity, comprehensibility, and changes they think should be done to improve the decision aid.

\section{Flow of the contents}

Both the HCPs and PAs said that the flow of the contents was good and was organized systematically and more simply.

"Generally the DA is good and the information in it covers the areas that we aspire to be covered in a FP counseling to be understood by every woman. The flow is good and I like the DA most because it involves pictures with interesting colors that will improve the clarity of the presented information" [HCP]

"To me, I see the information presented as normal as I cannot say it is too long or too short, the explanation is clear and easy to understand" [adolescent]

However, one HCP participant suggested a small significant change to be made in order to improve the logical flow of the contents. This participant said:

"I have a small correction. In the second step where the client is told to choose the reasons for selecting that option and then below that she is asked to choose the method (option). In my opinion, I thought that after the explanation of how each option works, the benefits and side effects, she should choose the option first then start to give the reasons why she thought maybe she chose that method (option)" [HCP]

The HCPs further said the contents included most of the critical information needed when providing counseling and key information/components important to aid decision-making. This provider said:

"Looking at the experience of counseling, there are lots of important aspects presented and often are the things clients ask especially [about] the effectiveness and fertility return. It [DA] has also described each option, where it is inserted, and how it works to prevent pregnancy" [HCP]

\section{Clarity of the information}

Language and medical terms used in the presentation were reported to be appropriate by both the HCPs and pregnant adolescents. The adolescents said the language used (Kiswahili) was clear, and everyone who gives it time to read will understand because the information was elaborated.

"I would say that the information presented in the tool [DA] was easy to understand because the Kiswahili words used are simple and well elaborated" [adolescent]

The HCPs said Kiswahili is a National language and that almost everyone in the country speaks it therefore the terminology used in this DA will be understood. Nevertheless, the HCPs gave some suggestions so as to reduce ambiguity like the use of Kitanzi instead of Lupu or use both, use of kichocheo instead of the "hormone", "slows down sperm motility" instead of "killing sperms", etc.

"There are some words that I thought if used would enhance clarity. For example, FP clients are [more] familiar with the word "kitanzi" than "LUPU". Use Kichocheo and not hormone as that is an English word. Similarly, killing sperm will be strange to women instead, rephrase it to "slow down the sperm motility" [HCP]

\section{Comprehensibility}

When the participants were further asked to give their opinion if the PAs could be given the DA to read by themselves, the HCPs said that the PAs would need support from providers to facilitate understanding of the information presented in pictures with colored and uncolored pictures as they believe that the PAs would not understand. In addition, Tanzanians do not have a culture of self-reading, especially with adolescents. So if the HCPs give them the DA to read by themselves it will be a challenge as they are not sure if the PAs will really read the tool. The HCPs advised that the information be read to all study participants who will be involved in the study.

"Mhhhh! It will be a challenge to read by themselves as we do not have a culture of self-reading. They might fail to appreciate the difference between the colored and the uncolored images. I recommend the HCPs to brief the participants about the information in every step and the 
meaning before they take the document home for further self-reading due to differences in the level of understanding" [HCP]

The PAs did not differ from what the HCPs suggested about self-reading of the DA. Almost all of the PAs said that it would be hard for them to read the DA by themselves. They requested someone to take them through the information presented before they are told to read by themselves.

"It will be difficult for me to read and understand all the information. When I finish reading the first page and go to the next page I do not remember what I read on the previous page" I need someone to explain to me as when I hear someone talking it sticks in my head more than if I read myself "[adolescent]

\section{Usefulness}

The study participants were asked to say if the DA: would improve knowledge, would address the existing myths and misconceptions in the community about LARFP methods that hinder its utilization, and if the benefits and side effects of each option are well clarified.

\section{a) Improves knowledge}

The PAs reported that the DA changed/improved their knowledge after reading. They said they did not know the existence of IUCDs and implants as well as the immediate postpartum FP methods. They did not even know if someone could use FP methods just after the birth of the placenta or before one month after childbirth. They further said that they did not know how the methods were put in place but now they could imagine it from the pictures shown in the DA.

"I did not have prior information about IUCDs and implants apart from pills and injectables but after reading, I realized there are other FP methods that even last longer than a year" [adolescent]

The HCPs affirmed that the decision aid would be useful for PAs. They further said the flow of the information is so clear and it explains the types of women, including adolescents, who can use any of the options, which is missing in most of the FP counseling sessions and guides.

"The tool [DA] is a narrative that can be read within a short period of time and be understood than reading the entire FP book. The tool pointed out people who can use LARFP methods that also included women of any age as long as they are sexually active. Most teenagers think that contraceptives are for adults above their twenties. So this information opens up their mind and helps them realize FP is for any woman regardless of age as long as she is sexually active. This part has been overlooked in our FP counseling even in FP clinical guides" [HCP]

\section{b) Adequately addresses the most common existing myths and misconceptions}

The HCPs reported having seen some myths and misconceptions addressed in the DA. However, they pointed out they are too few while in the community there are a lot of existing misbeliefs about FP methods, especially about LARFP methods. They pointed out misbeliefs such as, if women happened to conceive while the IUCD was in the uterus, when the baby is born, it would be holding the IUCD, or that IUCD and implants at times escape from where they are and go to the heart or brain. The HCPs further reported that there was no specific section that talks about myths and misconceptions. By just reading the DA alone, it would not be enough to dispel existing misbeliefs about FP methods for PAs. When the PAs were probed about this part they all kept quiet despite clarifying the question for them several times.

"The tool has addressed some of the existing myths and misconceptions indirectly though they are very few despite the fact that there are a lot of misbeliefs existing in our communities. If you address more of them this tool [DA] will be useful in addressing the existing misbeliefs" $[\mathrm{HCP}]$

"The information provided in this tool focused on the use of LARFP methods. But there is no specific section that addressed myths and misconceptions specifically. However, I see some of the misbeliefs in the tool like they do not cause uterine cancer, cannot interfere with the sexual act" [HCP]

One of the HCPs noted that the last part of the DA that requires the PA to note down some questions that need more clarification in the next antenatal clinic. That would help to explain more about the concerns and might also include myths and misconceptions she had about FP methods that were not explained in the DA. This HCP further said that it was not easy to address all of the myths and misconceptions existing in the community about IUCDs and implants as the DA would be too long. 
"Self-reading of the tool [DA], may not be so easy to correct the existing myths and misconceptions known by the participant. The part that might help to cover the question about myths and misconceptions is at the comments section. If she has any ideas or any misconceptions about the things she read and needs clarifications, I think this part might help. If she will have any question, if she has something she understood differently, writing here for more clarification in the next antenatal clinic visits will help to address the concerns the woman has about FP" $[\mathrm{HCP}]$

\section{c) Adequately clarifies the benefits and side effects of each option}

The HCPs reported that the DA clarifies the benefits and side effects of each option. The safety of artificial FP was a concern and was one of the barriers to using FP methods. Since the DA addresses such concerns it would provide realistic expectations of side effects and later would improve voluntary decision making to utilize FP immediately after delivery.

"Yeah! The area where society is stuck is in understanding how contraceptives work, how they prevent pregnancy, and if real they or do not cause all the complications (myths and misconceptions) they hear in the communities. The tool described the benefits and side effects that can be caused by each option. I think this tool will be useful in improving the utilization of FP" [HCP]

"The tool has explained well by comparing the benefits and side effects of each option, for example, I have read that the loop does not add on body weight and neither can my partner feel it during intercourse because they reduce the length of the string and this was my biggest fear as I heard it from my sister" [Adolescent]

\section{Acceptability}

We asked the study participants if they would recommend the DA to encourage women including adolescents to use LARFP methods. Two reasons for accepting the DA came out from the analysis; a) decision aid improves knowledge, and b) decision aid is a standardized guide with evidence-based information

\section{a) Decision aid improves knowledge}

The PAs said the DA should be used as it helps them get information that they were ignorant of before about the existence of FP offered immediately after giving birth.

"Let it [tool] be there in the clinic so that we can use it as it helped me to understand the available postpartum FP methods offered immediately after birth. I am sure even my fellow women will find the same benefit" [adolescent]

\section{b) Decision aid a standardized guide with evidence-based information}

Generally, the HCPs supported the use of the DA to complement their FP counseling education. They commended the DA that it is simplified and included all-important evidence-based information mothers need to learn the importance of child-spacing especially PPFP. It will also be good for use as a standardized guide for FP specifically for LARFP methods offered immediately after childbirth.

"Yes! The tool is so simplified and it included only the important information that a mother needs to be aware of in order to make an informed choice of the LARFP options. Generally, the tool is good to be used for both mother and caregiver" [HCP]

Another HCP further said that she would recommend the use of the DA because their health facilities do not have a standardized guide to be used for FP counseling. As a result, she finds the FP counseling being offered to be too subjective as they do not have a well-structured tool apart from the WHO FP handbook for HCPS [41]. So, variations of the information are given to mothers simply because of the differences in knowledge, time, and attitude among HCPs. But the use of the postpartum Green Star family planning decision aid is formal and will enhance the objectivity of the information offered to everyone.

"I will support the use of the tool because the current FP health education being offered is too subjective as there is no a standardized guide for FP counseling. Since this is formal it will ensure the objectivity of counseling to everyone as mothers will receive the same information from different HCPs than when there is no a standardized guide" [HCP]

\section{Discussion}

We highlight the following main findings regarding the feasibility of using a postpartum Green Star family planning decision aid for teenage women. First, on the matter of the DA practicality, the length of the presentation was found to be just right, the time reported to accomplish reading the DA ranged from 20 to 60 minutes. The flow of the information was good with small significant changes suggested 
by the HCPs to improve the logical flow. The Kiswahili language and medical terms used were reported to be clear and well elaborated but small changes of some words were suggested. Both the PAs and the HCPs recommended that the DA should be designed to be delivered by the HCPs but not for self-reading by the PAs.

Second, as for the usefulness of the decision aid, the PAs reported that the DA improved their knowledge about the existence of FP methods that could be used immediately after childbirth. They had not been aware of the existence of IUCDs and implants until reading the DA. The HCPs said the DA dispels some existing myths and misconceptions about IUCDs and implants. Both the PAs and the HCPs stated that the DA clearly addressed the benefits and side effects of each option.

Third, about the acceptability of the DA, the HCPs embraced the DA as a useful tool for complementing the daily FP counseling offered. They further said the DA would improve the objectivity of the FP counseling offered about LARFP methods. The PAs said that the DA improved their knowledge about LARFP, which they did not know about until they had read the DA.

Our DA is a unique tool designed to address the significant gap in FP delivery programs in developing countries. We designed the DA to be used by PAs but we also included the HCPs because we also wanted to explore the practicality, usefulness, and acceptability of the DA as perceived by the HCPs in relation to their experiences from current counseling practices. While the DA is designed for the PAs, results show the DA to be acceptable by both the PAs and the HCPs. The HCPs believed the DA to be useful in complementing their daily FP counseling as it also provides objectivity for the counseling, especially on LARFP methods. However, the HCPs suggested that HCP providers use the DA to guide PAs in making an informed decision instead of leaving it for the PA to read alone. The PAs said that for them to understand all the information, the HCPs should take them through the DA. It would be hard for PAs to read on their own as they were sure they would not read or would just read part of the information or would not read at all as others could not read and write. Tanzanians are reported to have a poor tendency of individually reading i.e. reading culture is poor [42].

In Tanzania, there are several DAs commonly used in clinics to provide FP counseling to women such as the WHO Decision Making Tools $[33,43]$, Balanced Counseling Strategy [44], flipcharts and counseling cards. These tools are only designed to support HCPs in FP counseling and they are not meant to help clients make informed decisions. There are some FP interventions implemented in Tanzania for example Chaguo la Maisha project by the Management and Development for Health and Pathfinder International [45]. The project provided essential information and accessibility of various FP methods to women of reproductive age through the use of community mobilizers.

However, the intervention had an insufficient strategy of meeting adolescents below 18 years as well as PAs to help them make an informed decision to help them prevent subsequent unwanted pregnancies. Tanzania also had an effective mobile job-aid used to provide FP counseling but the aid was only designed to support community health workers in the provision of FP service and not to support mothers in making a decision [46]. The postpartum Green Star family planning decision aid will be the first tool for pregnant adolescents-centered material for those who will attend antenatal care services.

Currently, in Tanzania, health providers conduct group FP counseling using the WHO Family Planning Handbook [41] sometimes with the assistance of flipcharts and a FP medical eligibility criteria wheel [43]. The FP counseling offered to women is mainly provider dependent. The postpartum Green Star family planning decision aid will offer opportunities that ensure: 1) women receive the key information about LARFP methods as they are the only effective reversible methods recommended and available immediately after childbirth [17], and 2) the FP information is objectivity delivered to each PA.

Findings from this feasibility study suggest that our postpartum Green Star family planning decision aid is practical, useful, and acceptable for educating pregnant adolescents about LARFP methods available immediately after childbirth in Tanzania. To improve the quality of the DA some modifications were proposed like rearranging the flow of the information or remove one of the sections, change some of the Kiswahili words to remove ambiguity. It was also suggested that the DA should be delivered by the HCPs rather than letting PAs read by themselves in order to ensure comprehensibility of the information that would simplify the decision-making process. Adding content that corrects details found in the FP myths and misconceptions or omitting unimportant information is expected to further enhance the practicality, usefulness, and acceptability of the DA. Apparently, there are limited published studies that have researched the feasibility of any FP decision aid about LARFP methods with which to make a comparison with the findings from this present study.

\section{Strengths And Limitations Of The Study}

The study included the PAs and the HCPs to assess the practicality, usefulness, and acceptability of the postpartum Green Star family planning decision aid. The Ottawa Decision Support Framework guided the development of this DA and has been widely used in various clinical areas and proved to show positive outcomes regarding decision-making. The study participants were purposively selected to ensure the veracity and appropriateness of the decision aid. Our study had a few limitations as follows. The study participants came from Amana 
hospital, which is one of the several district hospitals in Dar es Salaam. Therefore, the findings cannot be used to generalize other settings or groups of adolescents.

\section{Conclusion}

The postpartum Green Star family planning decision aid was perceived by both the PAs and the HCPs to be feasible in terms of practicality, usefulness, and acceptability. Family planning counseling tools with evidence-based information can support PAs to improve knowledge hence improve an informed voluntary decision. Further research is needed to assess the effect of the postpartum Green Star family planning decision aid on choice for postpartum family planning among pregnant adolescents in Tanzania.

\section{Abbreviations}

WHO: World Health Organization; TDHS: Tanzania Demographic Health Survey; FP: family planning; RRP: rapid repeat pregnancy; LARFP: long-acting reversible family planning; IUCDs: intrauterine copper devices; PPFP: postpartum family planning: DA: decision aid; HCPs: healthcare providers; PI: principal investigator; RA: research assistant; MEC: medical eligibility criteria, PAs: pregnant adolescents

\section{Declarations}

\section{Ethics approval and consent to participate}

The study was approved by the Institutional Review Boards of St. Luke's International University (19-A078), Muhimbili University of Health and Allied Sciences and Tanzania National Institute of Medical Research. Written informed consent was provided by all participants prior to study participation.

\section{Consent for publication}

Study participants provided consent to publish the data.

\section{Availability of data and materials}

Not applicable

\section{Competing interests}

Authors do declare that there are no competing interests

\section{Funding}

The study was funded by the Japan Society for the Promotion of Science Core-to-core program (2018-2021).

\section{Acknowledgments}

We thank Zeswida Ahmed and Agnes F. Massae for their assistance in data collection and transcription of the data. Our appreciation goes to Gift Lukumay for his/her assistance with the translation of the data collection tools and the decision aid. Our sincere gratitude to the Japan Society for the Promotion of Science for the study sponsorship. Our special thanks to all respondents who participated in this study.

\section{Authors' contributions}

SEM; conceptualization, formal analysis, investigation, methodology, supervision, writing -original manuscript, writing-review \& editing

ES; conceptualization, supervision, visualization, validation, writing-review \& editing

SL; conceptualization, supervision, resources, formal analysis \& validation

SH; conceptualization, formal analysis, investigation, methodology, funding acquisition, supervision, resources, validation, writing-review \& editing

\section{References}


1. World Health Organization. Adolescent pregnancy: key facts (2018). https://www.who.int/news-room/fact-sheets/detail/adolescentpregnancy. Accessed July 2019.

2. Neal S, Matthews Z, Frost M, Fogstad H, Camacho AV, Laski L. Childbearing in adolescents aged 12-15 in low resource countries: a neglected issue. New estimates from demographic and household surveys in 42 countries. Acta Obstet Gynecol Scand, 2012;doi:10.1111/j.1600-0412.2012.01467.x.

3. Tanzania Demographic and Health Survey and Malaria Indicator Survey. Dar es Salaam, Tanzania. MoHCDGEC Ministry of Health CD, Gender, Elderly and Children - MoHCDGEC/Tanzania Mainland, MOH Ministry of Health - MoH/Zanzibar, NBS National Bureau of Statistics - NBS/Tanzania. OCGS Office of Chief Government Statistician - OCGS/Zanzibar, ICF. 2015 - 2016 https://dhsprogram.com/pubs/pdf/FR321/FR321.pdf. Accessed May 2019.

4. The United Republic of Tanzania. The National Road Map Strategic Plan II to Improve Reproductive, Maternal, Newborn, Child \& Adolescent Health in Tanzania. Ministry Of Health, Community Development, Gender, Elderly and Children. 2016 - 2020. https://www.globalfinancingfacility.org/sites/gff_new/files/Tanzania_One_Plan_Il.pdf. Accessed April 2019.

5. The United Republic of Tanzania. The National Family Planning Costed Implementation Program. Ministry of Health and Social Welfare. 2010-2015. http://ec2-54-210-230-186.compute-1.amazonaws.com/wpcontent/uploads/2014/10/NFPCIP_Amendment_NEW2.pdf. Accessed April 2019.

6. Population Reference Bureau. Tanzania Youth Reproductive Health: Satisfying an unmet need for family planning. 2015. https://assets.prb.org/pdf15/unmetneed-factsheet-tanzania.pdf. Accessed September 2019.

7. United Nations Population Fund. Adolescent Pregnancy: A Review of the Evidence. (2013). https://www.unfpa.org/sites/default/files/pub-pdf/ADOLESCENT\%2OPREGNANCY_UNFPA.pdf. Accessed June 2019.

8. Briggs G, Brownell M, Ross N. Teen mothers and socioeconomic status. The chicken egg debate. Journal of the Motherhood Initiative for Research and Community Involvement. 2007. https://jarm.journals.yorku.ca/index.php/jarm/article/view/5136/4332. Accessed May 2019.

9. Jones ME, Mondy LW. Lessons for prevention and intervention in adolescent pregnancy: A five year comparison of outcomes of low programs for school aged pregnant adolescents. Journal of Pediatric Health Care. 1994; doi: 10.1016/0891-5245(94)90027-2.

10. Mushy SE, Tarimo EAM, Fredrick MA, Horiuchi S. Barriers to the uptake of modern family planning methods among female youth of Temeke District in Dar es Salaam, Tanzania: A qualitative study. Sex Reprod Healthc. 2020; doi:10.1016/j.srhc.2020.100499.

11. Darroch JE, Woog V, Bankole A, Ashford LS. Adding It Up: Costs and Benefits of Meeting the Contraceptive Needs of Adolescents, New York: Guttmacher Institute. 2016. https://www.guttmacher.org/report/adding-it-meeting-contraceptive-needs-of-adolescents_.Accessed April, 2019.

12. Cleland J, Conde-Agudelo A, Peterson H, Ross J, Tsui A. Family planning needs during the first two years postpartum in Tanzania. Contraception and health. The Lancet. 2012; 380(9837):149-156. http://reprolineplus.org/system/files/resources/Tanzania-PPFP.pdf. Accessed April 2019.

13. Oringanje C, Meremikwu MM, Eko H, Esu E, Meremikwu A, Ehiri JE. Interventions for preventing unintended pregnancies among adolescents. Cochrane Database of Systematic Reviews. 2016;doi:10.1002/14651858.CD005215.pub3.

14. Alford S, Rutledge A, Huberman B. Science and success. Program that works to prevent subsequent pregnancy among adolescent mothers. Adolescent for youth. 2014. https://www.advocatesforyouth.org. Accessed November 2020.

15. De Jonge HCC, Azad K, Seward N, Kuddus A, Shaha S, Costello A, Houweling TAJ, Fottrell E, Beard J. Determinants and consequences of short birth interval in rural Bangladesh: a cross-sectional study. BMC Pregnancy and Childbirth. 2014; doi: 10.1186/s12884-0140427-6.

16. Primary health care: Report of the international conference on primary health care. Alma-Ata, USSR, $6-12$ September 1978. Jointly sponsored by the World Health Organization and the United Nations Children's Fund. http://who.int/medicinedocs/en/m/abstract/Js21370en/_Accessed May 2019.

17. The American College of Obstetricians and Gynecologists. Long-acting reversible contraception: implants and intrauterine devices. Obstet Gynecol. 2017; 130:251-69. Retrieved from: https://www.acog.org/-/media/Practice-Bulletins/Committee-on-Practice-Bulletins-Gynecology/Public/pb186.pdf. Accessed May 2019.

18. Baldwin MK, Edelman AB. The Effect of Long-Acting Reversible Contraception on Rapid Repeat Pregnancy in Adolescents: A Review. Journal of Adolescent Health: 2013; 52: S47eS53. https://www.jahonline.org/article/S1054-139X(12)00715-X/pdf. Accessed June 2019.

19. Stacey D, Légaré F, Lewis K, Barry MJ, Bennett CL, Eden KB, Holmes-Rovner M, Llewwllyn-Thomas H, Lyddiatt A, Thomson R, Trevena L. Decision aids for people facing health treatment or screening decisions. Cochrane Database of Systematic Reviews-Intervention. 
2017;doi:10.1002/14651858.CD001431.pub5.

20. Bennett KF, Wagner VC, \& Robb KA. Supplementing factual information with patient narratives in the cancer screening context: a qualitative study of acceptability and preferences. 2015;doi:10.1111/hex.12357.

21. Wu JP, Damnshroder LJ, Fetters MD, Zikmund-Fisher BJ, Hudson SV, Fucinari JBS, Taichman LS, Creswell JW. A Web-Based Decision Tool to Improve Contraceptive Counseling for Women with Chronic Medical Conditions: Protocol for a Mixed Methods Implementation Study. JMIR Res Protoc. 2018; doi:10.2196/resprot.9249.

22. Kim YM, Davila C, Tellez C, Kols A. Evaluation of the World Health Organization's family planning decision-making tool: improving health communication in Nicaragua. Patient Education and Counseling. 2007;doi:10.1016/j.pec.2006.12.007.

23. Braun V, Clarke V. Thematic analysis. In H. Cooper, P. M. Camic DL, Long AT, Panter D Rindskopf, K J Sher (Eds.), APA handbooks in psychology ${ }^{\circledR}$. APA handbook of research methods in psychology. Research designs: Quantitative, qualitative, neuropsychological, and biological. American Psychological Association. 2012; 2: 57-71.

24. Cascio MA, Lee E, Vaudrin N, Freedman DA. A Team-based Approach to Open Coding: Considerations for Creating Intercoder Consensus. Field Method. 2019; doi: 10.1177/1525822X19838237.

25. Shishindo E, Osaka W, Henna A, Motomura Y, Horiuchi S. Effect of a decision aid on the choice of pregnant women whether to have epidural anesthesia or not during labor. PLoS One. 2020;doi:10.1371/journal.phone.0242351.

26. Osaka W, Nakayama K. Effect of a decision aid with patient narratives in reducing decisional conflict in choice for surgery among earlystage breast cancer patients: A three-arm randomized controlled trial. Patient Education and Counseling. 2017; doi:10.1016/j.pec.2016.09.011.

27. Brown S, Lumley J. Satisfaction with care in labor and birth: A survey of 790 Australian women. Birth Issues in Prenatal Care. 1994; doi: 10.1111/j.1523-536X.1994.tb0009.x.

28. Ottawa Patient Decision Aid Development eTraining (ODAT). Patient Decision Aids. https://decisionaid.ohri.ca/index.html. Accessed August 2019.

29. The International Patient Decision Aid Standards (IPDAS) Collaboration. IPDAS Criteria for Judging the Quality of Patients Decision Aids. 2005. http://ipdas.ohri.ca/IPDAS_checklist.pdf. Accessed June 2019.

30. Ajzen I. The theory of planned behavior. Organizational Behavior and Human Decision Processes; 1991; doi: 10.1016/07495978(91)90020-T.

31. Green EC, Murphy E. Health belief model. In The Wiley Blackwell encyclopedia of health, illness, behavior, and society. 2014;doi:10.1002/9781118410868.wbehibs410.

32. Bandura A. Social cognitive theory. Handbook of social psychological theories. 2011;doi:doi.10.4135/9781446249215.n18.

33. World Health Organization (WHO) and Johns Hopkins Bloomberg School of Public Health. Center for Communication Programs. Information and Knowledge for Optimal Health (INFO). Decision-making tool for family planning clients and providers. Baltimore, Maryland, INFO and Geneva, WHO. (WHO Family Planning Cornerstone). 2015.

https://apps.who.int/iris/bitstream/handle/10665/43225/9241593229_eng.pdf;jsessionid=9D552FBA2D0A7F09857A01137127E5FE? sequence=2. Accessed October 2020.

34. Peipert JF, Zhao Q, Allsworth JE, Petrosky E, Madden T, Eisenberg D, Secura G. Continuation and Satisfaction of Reversible Contraception. Obstetric and Gynecology. 2011; doi:10.1097/AOG.0b014e31821188ad.

35. Yisa SB, Okenwa AA, Husemeyer RP. Treatment of pelvic endometriosis with etonogestrel subdermal implant (Implanon $\left.{ }^{\circledR}\right)$. J Fam Plann Reprod Health Care. 2005; doi: 10.1783/0000000052972799.

36. Funk S, Miller MM, Mishell DR, Archer DF, Poindexter A, Schmidt J, Zampaglione E. Safety and efficacy of Implanon, a single-rod implantable contraceptive containing etonogestrel. Contraception. 2005;doi:10.1016/j.contraception.2004.11.0007.

37. Hubacher D, Grimes DA. Non-contraceptive health benefits of intrauterine devices: a systematic review. Obstet Gynecol Surv. 2002; 57(2):120-8. https://www.ncbi.nlm.nih.gov/pubmed/11832788. Accessed May 2019.

38. Zheng SR, Zheng HM, Qian SZ, Sang GW, Kaper RF. A randomized multicenter study comparing the efficacy and bleeding pattern of a single - rod (Implanon) and a six-capsule (Norplant) hormonal contraceptive implant. Contraception. 1999; doi: 10.1016/S00107824(99)00053-0.

39. Soeprono R. Return to fertility after discontinuation of copper IUD use: a study of 55 pregnancies involving Multiload Cu-250 users among private patients in Indonesia. Advances in Contraception. 1988; doi: 10.1007/BF01849510.

40. Buckshee K, et al. Return of fertility following discontinuation of Norplant-Il subdermal implants: ICMR task force on hormonal contraception. 1995; doi: 10.1016/0010-7824(95)00039-D.

Page 12/13 
41. World Health Organization Department of Reproductive Health and Research (WHO/RHR) and Johns Hopkins Bloomberg School of Public Health/Center for Communication Programs (CCP), Knowledge for Health Project. Family Planning: A Global Handbook for Providers (2018 update). Baltimore and Geneva: CCP and WHO, 2018. https://www.who.int/reproductivehealth/publications/fp-globalhandbook/en/. Accessed September 2019

42. Masabo CJ. The Declining of the Reading Culture in Tanzania: A Case of the Institute of Adult Education. LAP LAMBERT Academic Publishing. 2015. https://www.amazon.com/Declining-Reading-Culture-Tanzania-Institute/dp/3659779334._Accessed November 2020.

43. World Health Organization. Medical eligibility criteria for contraceptive use. Fifth edition, 2015. http://apps.who.int/iris/bitstream/10665/181468/1/9789241549158_eng.pdf. Accessed November 2020.

44. Leon FR, Vernon R, Martin A, Bruce L. The balanced counseling strategy; a toolkit for family planning service providers. Washington, DC: Population Council. 2008. http://citeseerx.ist.psu.edu/viewdoc/download?doi=10.1.1.175.8134\&rep=rep1\&type=pdf. Accessed October 2020.

45. Komwihangiro J, Mwandalima I, Stephens B, Temba A. Mainstreaming youth friendly services in public health facilities in urban Tanzania: Chaguo la Maisha Project. 2018. https://dqo52087pnd5x.cloudfront.net/posters/docs/gatesopenres-185203.pdf. Accessed November 2020.

46. Brawn R, Lasway C, Agarwal S, L'Engle K, Layer E, Silas L, Mwakibete A, Kudrati M. An evaluation of a family planning mobile job aid for community health workers in Tanzania. An International Reproductive Health Journal. Contraception. 2016; doi:10.1016/j.contraception.2016.03.016.

\section{Figures}

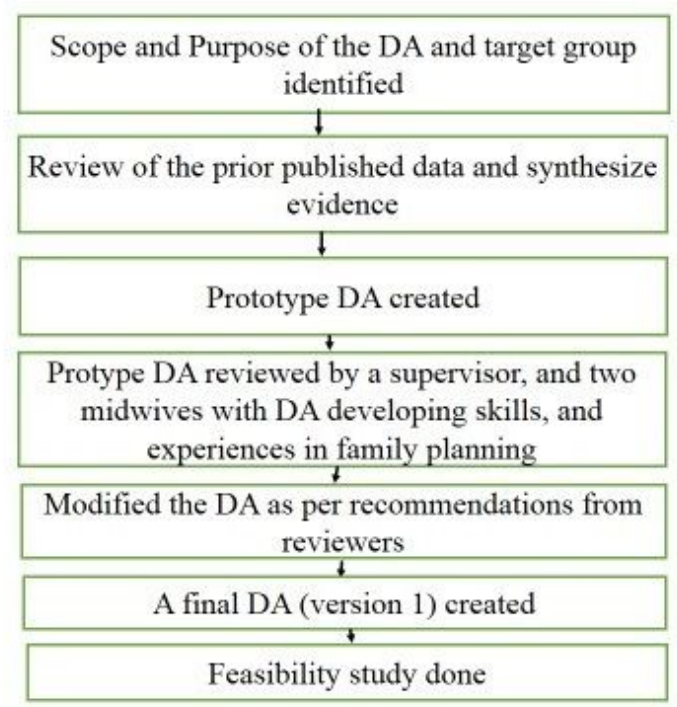

\section{Figure 1}

Flow diagram showing the process of developing the postpartum Green Star family planning decision aid. A final decision aid version 1 